\title{
Estrutura dimensional da satisfação dos usuários da Saúde da Família
}

Eliana Campêlo Lago ${ }^{1}$, Fabricio Ibiapina Tapety ${ }^{2}$, Camila Aparecida Pinheiro Landim Almeida ${ }^{3}$, Matheus Costa Brandão Matos ${ }^{4}$, Laelson Rochelle Milanes Sousa ${ }^{5}$, Maria do Carmo de Carvalho e Martins ${ }^{6}$, Adélia Dalva da Silva Oliveira ${ }^{7}$, Luana Kelle Batista Moura ${ }^{8}$, Luciana Barros de Moura Neiva ${ }^{9}$

\section{RESUMO}

O estudo objetiva analisar a estrutura dimensional do instrumento SERVQUAL, utilizado para mensuração da satisfação dos usuários de serviço de saúde pública na atenção básica. Estudo quantitativo, realizado por meio de um survey com 353 usuários da Estratégia Saúde da Família. Os participantes foram: gênero feminino (71,7\%); idades entre 31 e 40 anos (30,9\%) e com Ensino Médio completo (43,3\%). Na análise do ajuste do modelo, os resultados foram insatisfatórios para os critérios estabelecidos. Sugeriu-se um novo modelo dado o contexto específico da saúde pública que apresentou resultados adequados para todos os critérios. Os resultados foram satisfatórios para validade convergente e discriminante. O estudo implica na indicação de um novo modelo ajustado, utilizado para a compreensão da satisfação de usuários da saúde da família, que, testado em estudos futuros na área de enfermagem e saúde pública, com análise aprofundadas que discutam os achados desta pesquisa, consolidem um novo instrumento analítico.

Descritores: Satisfação do Paciente; Estratégia Saúde da Família; Atenção Primária à Saúde.

\footnotetext{
${ }^{1}$ Enfermeira, Doutora em Biotecnologia. Coordenadora do Programa de Mestrado Profissional em Saúde da Família do Centro Universitário UNINOVAFAPI. Teresina, PI, Brasil. E-mail: eliana@uninovafapi.edu.br.

${ }^{2}$ Cirurgião-Dentista, PhD em Clinical Dentistry. Professor do Centro Universitário UNINOVAFAPI. Teresina, PI, Brasil. E-mail:

ftapety@uninovafapi.edu.br.

${ }^{3}$ Enfermeira, Doutora em Ciências. Professora do Centro Universitário UNINOVAFAPI. Teresina, PI, Brasil. E-mail: camila@uninovafapi.edu.br.

${ }^{4}$ Discente do curso de graduação da Universidade Federal do. Teresina, PI, Brasil. E-mail: matheuscbmatos@gmail.com.

${ }^{5}$ Enfermeiro, Mestre em Enfermagem. Discente do Programa de Pós-Graduação em Enfermagem Fundamental, nível Doutorado, da Escola de Enfermagem de Ribeirão Preto da Universidade de São Paulo. Ribeirão Preto, SP, Brasil. E-mail: laelson@usp.br.

${ }^{6}$ Nutricionista, Doutora em Ciências Biológicas. Professor Titular da Universidade Federal do Piauí. Teresina, PI, Brasil. E-mail:

carminhamartins@ufpi.edu.br.

${ }^{7}$ Enfermeira, Doutora em Políticas Públicas. Coordenador do curso de Enfermagem do Centro Universitário UNINOVAFAPI. Teresina, PI, Brasil. Email: oliveiracairo@ig.com.br.

${ }^{8}$ Cirurgiã-Dentista, Doutora em Endodontia. Professora do Centro Universitário UNINOVAFAPI. Teresina, PI, Brasil. E-mail:

luanamoura@uninovafapi.edu.br.

${ }^{9}$ Enfermeira, Doutora em Enfermagem na Saúde do Adulto. Professora da Faculdade Estácio de Teresina. Teresina, PI, Brasil. E-mail: lucianabmneiva@uol.com.br.
}

Artigo recebido: 06/01/2018.

Artigo aprovado: 17/10/2018.

Artigo publicado: 31/12/2018.

\section{Como citar esse artigo:}

Lago EC, Tapety FI, Almeida CAPL, Matos MCB, Sousa LRM, Martins MCC, et al. Estrutura dimensional da satisfação dos usuários da Saúde da Família. Rev. Eletr. Enf. [Internet]. 2018 [acesso em:

];20:v20a55. Disponível em: https://doi.org/10.5216/ree.v20.51007. 


\section{INTRODUÇÃO}

O Sistema Único de Saúde (SUS) é estruturado em diferentes níveis de atenção à saúde que se organizam de acordo com o nível de complexidade. A Atenção Básica (AB) é caracterizada como a principal porta de entrada ao sistema e é responsável pela saúde das famílias e populações de difícil acesso por meio da Estratégia Saúde da Família (ESF). A ESF foi implantada no Brasil como principal estratégia de reorganização da $A B$, é alocada em regiões estratégicas para facilitar o acesso da população aos serviços do SUS ${ }^{(1)}$.

Para que a população tenha acesso aos serviços da ESF de forma consistente conforme suas necessidades, é necessário que se crie vínculo entre profissionais de saúde e usuários dos serviços. Os vínculos estabelecidos aumentam a confiança e satisfação dos usuários ${ }^{(1)}$. A satisfação na área da saúde pode ser encarada como um processo complexo relacionado às necessidades e expectativas individuais, envolvendo diferentes aspectos de atenção. Dessa forma, a satisfação consiste em uma avaliação de diferentes elementos dos cuidados de saúde, como infraestrutura, acesso e qualidade dos recursos humanos, ocupando uma posição diferenciada nos processos de avaliação dos serviços ${ }^{(2)}$.

Uma vez que a satisfação do usuário interfere no nível de adesão terapêutica e nos comportamentos frente à saúde e doença, sua medição é indispensável para a avaliação de políticas públicas e dos serviços de saúde no que diz respeito à qualidade do serviço e capacidade de resposta do sistema. Em contextos internacionais, nos países que tratam a saúde como mercadoria e o usuário como consumidor, o aumento da satisfação implica em aumento do consumo dos serviços de saúde ${ }^{(3)}$. No contexto brasileiro, em serviços públicos de saúde, vinculados ao SUS, a satisfação se associa às melhorias do estado de saúde, envolvendo o paciente de forma ativa nas decisões tomadas acerca de sua assistência ${ }^{(1)}$.

A Estratégia Saúde da Família (ESF) prioriza ações de proteção e promoção à saúde dos indivíduos e da família, reiterando os princípios básicos do Sistema Único de Saúde (SUS) e substituindo o modelo tradicional biomédico. Entretanto, nota-se que o nível de satisfação do usuário é decisório na procura pelos serviços ${ }^{(4)}$. 0 conhecimento destes níveis pode balizar as ações e o desenvolvimento de novos programas e estratégias, uma forma pontual de identificar falhas e contribuir para a melhoria da qualidade dos serviços.

Visto que a avaliação do atendimento oferecido subsidia a melhoria do sistema de saúde, e que a participação livre e crítica dos usuários favorece o empoderamento dentro do sistema, este estudo objetivou analisar a estrutura dimensional do instrumento SERVQUAL, utilizado para a mensuração da satisfação dos usuários de serviço de saúde pública na atenção básica. A escala SERVQUAL ou Service Quality caracteriza-se como um instrumento estatístico bastante utilizado para a mensuração da qualidade de serviços a partir da percepção de seus usuários ${ }^{(5)}$. Ao analisar os gaps ou discrepâncias que interferem no nível de qualidade de serviços oferecidos ao usuário, a escala aponta a divergência existente entre as percepções e expectativas, analisando a maneira como o serviço é ofertado ao usuário e levantando os aspectos que precisam ser melhorados dentro de uma instituição(6).

A avaliação da satisfação dos usuários de serviços de saúde ainda de participação diminuta na literatura acadêmica, especialmente possibilitada por meio da aplicação da escala SERVQUAL, pode contribuir com o potencial inovador do uso de instrumentos de gestão convertidos em instrumentos analíticos da saúde pública a partir da perspectiva de seus usuários. 


\section{MATERIAL E MÉTODO}

\section{Aspectos éticos}

O projeto de pesquisa foi aprovado pelo Comitê de Ética e Pesquisa do Centro Universitário UNINOVAFAPI sob parecer no 893.065 e CAAE no 38532614.0.0000.5210. Os participantes da pesquisa assinaram o Termo de Consentimento Livre e Esclarecido. Foi garantida a confidencialidade dos participantes.

\section{Delineamento e dados do estudo}

Empreendeu-se um estudo quantitativo, realizado por meio de um survey com 353 usuários da Estratégia Saúde da Família que eram atendidos na cidade de Teresina/PI (Brasil). A coleta de dados ocorreu de janeiro a maio de 2015. A pesquisa foi realizada com usuários de duas equipes da ESF na zona leste da cidade. No momento da pesquisa, 927 famílias eram beneficiadas pelas duas equipes que compunham a ESF investigada. Para além da avaliaçãodo nível de satisfação dos usuários, indicação geral de uso da escala, o estudo pretendeu analisar suas dimensões para o contexto específico da saúde pública a partir da sua aplicação.

\section{População e amostra: critérios de inclusão e exclusão}

Foram seguidos os seguintes critérios de inclusão: estar cadastrado na ESF; ter idade igual ou superior a 18 anos; ser capaz de responder ao questionário e ter sido atendido no mínimo uma vez por uma das equipes da ESF. O critério de exclusão utilizado era ser funcionário da ESF ou de qualquer outro serviço ligado à Fundação Municipal de Saúde de Teresina.

Para estimar a população de indivíduos atendidos, foi utilizada a média de pessoas por família em Teresina, calculada conforme dados do último censo realizado pelo Instituto Brasileiro de Geografia e Estatística (IBGE) realizado em 2010 e de acordo com estimativas atuais do ano de 2018 (média de 3,65 pessoas) com estimativa de 3.384 pessoas atendidas ${ }^{(7)}$. A partir da estimativa da população atendida, dados que apoiaram o cálculo para determinação do tamanho da amostra, utilizou-se fórmula apresentada na Fórmula 1 para determinação amostral, o que guiou a quantidade de questionários aplicados.

Fórmula 1: Cálculo da amostra

$$
\mathrm{n}=\frac{\mathrm{z}^{2} \mathrm{xPxQxN}}{\mathrm{e}^{2} x(\mathbb{N}-1)+z^{2} \times P \times Q}
$$

Nota:

- $\quad Z$ = nível de confiança (95\%).

- $P=$ quantidade de acerto esperado (50\%).

- $Q=$ quantidade de erro esperado (50\%).

- $\mathrm{N}=$ população (3.384 pessoas).

- $\quad$ e = nível de precisão (5\%).

- $\mathrm{n}=345$. 


\section{Instrumento e coleta de dados}

O instrumento de coleta de dados da pesquisa está dividido em duas seções. A primeira seção contém os dados sociodemográficos. Na segunda são apresentadas as afirmações utilizadas para mensurar os construtos. As questões correspondem às cinco dimensões da qualidade, conforme modelo SERVQUAL adaptado, também utilizado em outros estudos mais recentes ${ }^{(8-9)}$. As dimensões investigadas correspondem à confiabilidade (Variáveis P1 a P5), presteza (P6 a P9), segurança (P10 a P13), empatia (P14 a P18) e tangibilidade (P19 a P22) (Quadro 1). As opções de resposta são graduadas em escala Likert, para medir o nível de concordância em relação às afirmações, variando de "concordo totalmente" a "discordo totalmente", com cinco pontos.

Quadro 1: Cinco dimensões de avaliação propostas pelo modelo SERVQUAL.

\begin{tabular}{|c|c|}
\hline Construto & Variáveis \\
\hline \multirow{5}{*}{$\begin{array}{l}\text { Confiabilid } \\
\text { ade }\end{array}$} & P1. Quando a estratégia saúde da família da localidade promete fazer um serviço em certo tempo, ela o faz. \\
\hline & $\begin{array}{l}\text { P2. Quando você tem algum problema de saúde, a estratégia saúde da família da localidade demonstra o sincero } \\
\text { interesse em resolvê-lo. }\end{array}$ \\
\hline & P3. A estratégia saúde da família da localidade faz o serviço certo da primeira vez. \\
\hline & P4. A estratégia saúde da família da localidade presta os serviços na data prometida. \\
\hline & P5. A estratégia saúde da família da localidade insiste em registrar os atendimentos realizados. \\
\hline \multirow{4}{*}{ Presteza } & $\begin{array}{l}\text { P6. Funcionários da estratégia saúde da família da localidade dizem exatamente quando os serviços de saúde serão } \\
\text { executados }\end{array}$ \\
\hline & P7. Funcionários da estratégia saúde da família da localidade fornecem um serviço imediato a você. \\
\hline & P8. Os funcionários da estratégia saúde da família da localidade estão sempre dispostos a ajudá-lo. \\
\hline & $\begin{array}{l}\text { P9. Empregados da estratégia saúde da família da localidade nunca estão muito ocupados para atender as suas } \\
\text { procuras por atendimento. }\end{array}$ \\
\hline \multirow{4}{*}{ Segurança } & $\begin{array}{l}\text { P10. O comportamento dos funcionários da estratégia saúde da família da localidade gera confiança em você, em } \\
\text { relação ao serviço realizado. }\end{array}$ \\
\hline & P11. Você se sente seguro em solicitar serviços da estratégia saúde da família da localidade. \\
\hline & P12. Funcionários da estratégia saúde da família da localidade são sempre educados com você. \\
\hline & $\begin{array}{l}\text { P13. Os funcionários da estratégia saúde da família da localidade têm o conhecimento para responder às suas dúvidas, } \\
\text { a respeito dos serviços de saúde. }\end{array}$ \\
\hline \multirow{5}{*}{ Empatia } & P14. Funcionários da estratégia saúde da família da localidade dão atenção individual a você. \\
\hline & P15. A estratégia saúde da família da localidade tem horário conveniente para atendê-lo em consultas. \\
\hline & P16. A estratégia saúde da família da localidade tem funcionários que dão atenção pessoal a você. \\
\hline & P17. Os funcionários da estratégia saúde da família da localidade entendem suas necessidades. \\
\hline & $\begin{array}{l}\text { P18. Os funcionários da estratégia saúde da família da localidade compreendem as suas necessidades específicas de } \\
\text { saúde. }\end{array}$ \\
\hline \multirow{4}{*}{$\begin{array}{l}\text { Tangibilida } \\
\text { de }\end{array}$} & P19. O posto de saúde da estratégia saúde da família da localidade tem equipamentos modernos. \\
\hline & P20. As instalações físicas do posto de saúde da estratégia saúde da família da localidade são visualmente atrativas. \\
\hline & P21. Os empregados do posto de saúde da estratégia saúde da família da localidade são bem vestidos e asseados. \\
\hline & $\begin{array}{l}\text { P22. A aparência das instalações físicas do posto de saúde da estratégia saúde da família da localidade é conservada } \\
\text { de acordo com o serviço oferecido. }\end{array}$ \\
\hline
\end{tabular}

Fonte: Elaborado pelos autores a partir da escala original adaptada(17).

\section{Análise dos resultados e estatística}

A análise estatística foi realizada através do software Statistical Package for the Social Sciences (SPSS), em que foram usados módulos de estatística descritiva. Para a Análise Fatorial Confirmatória do modelo proposto (Figura 1) foi usado o complemento do software SPSS Analysis of Moment Structures (AMOS). 
Figura 1: Modelo da Análise Fatorial Confirmatória, Teresina, PI, Brasil, 2016.

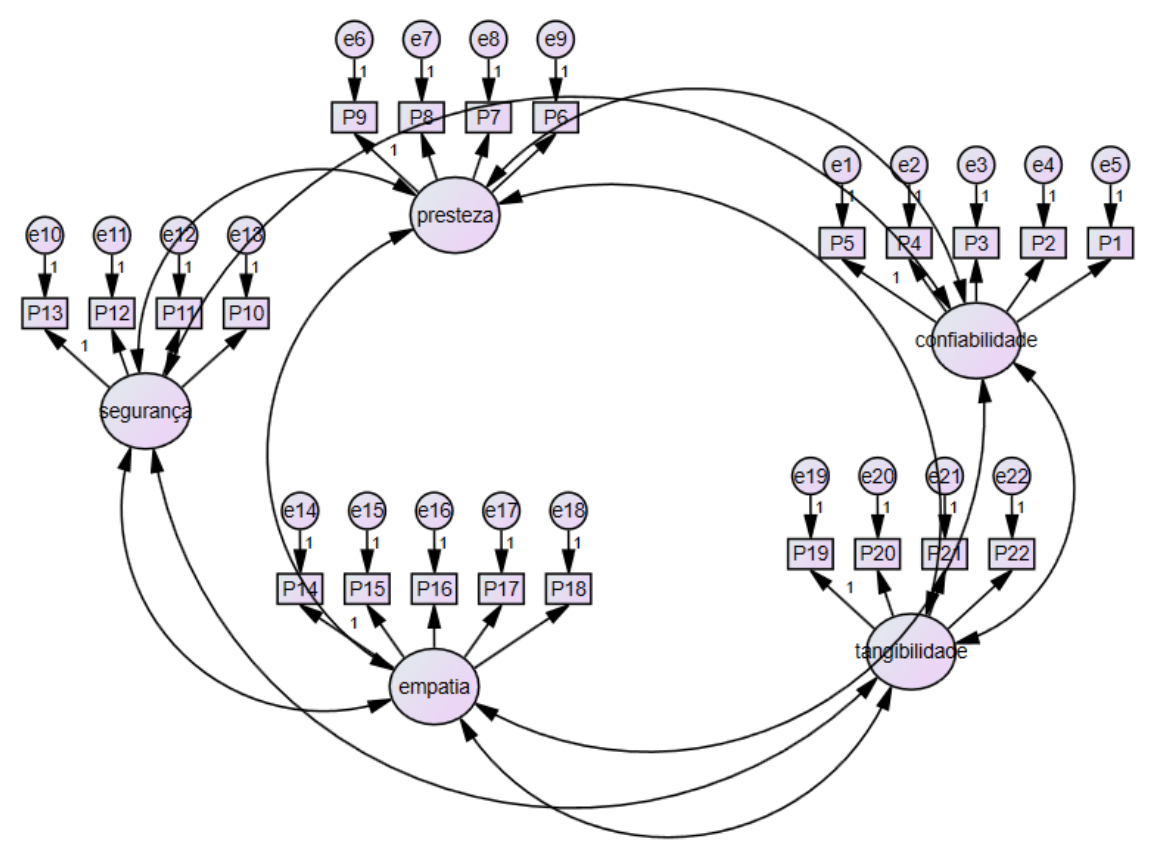

\section{Análise multivariada}

Quanto à análise do modelo foram utilizados os seguintes critérios, cujos valores correspondem ao padrão utilizado e recomendado pela literatura para análises deste tipo: GFI (índice de qualidade de ajuste) e CFI (índice de ajuste comparativo) superiores a 0,90; CMIN/DF (Qui-quadrado relativo) inferior a cinco e o RMSEA (raiz do erro quadrático médio de aproximação) inferior a 0,08, para ajuste do modelo. Para validade convergente utilizousedo Alfa de Cronbach e do CR (Índice composto de confiabilidade) acima de 0,7, do AVE (variância média extraída) acima de 0,5 e para validade discriminante o AVEmaior do que o MSV (variância máxima compartilhada) e do ASV (variância média compartilhada) $)^{(10-12)}$.

\section{RESULTADOS}

\section{Análise descritiva}

Os participantes da pesquisa apresentaram a seguinte caracterização: 253 (71,7\%) do gênero feminino; de idades entre 31 e 40 anos (30,9\%); casados (46,7\%); e a escolaridade era Ensino Médio completo (43,3\%); a renda familiar de $64,1 \%$ da amostra variava entre um e dois salários mínimos com 58,9\% de desempregados.

\section{Análise multivariada}

$\mathrm{Na}$ análise do ajuste do modelo os resultados foram insatisfatórios para os critérios estabelecidos: GFI = 0,$849 ; \mathrm{CFI}=0,864 ; \mathrm{CMIN} / \mathrm{DF}=3,700 ;$ e RMSEA $=0,088$. Quanto à validade convergente e discriminante, o modelo apresentou problemas para validade convergente com os construtos presteza $(0,486)$ e segurança $(0,478)$ apresentando valores menores do que 0,5 .

Desta forma, sugeriu-se um novo modelo, em que foram retiradas as variáveis P1, P6, P12 e P13. Supõe-se que essas variáveis não se ajustaram ao construto devido aos seus enunciados, a P1 e P6 falam sobre tempo, algo 
fora do escopo do construto. Já, a P12 e P13 não se adequaram ao construto segurança, pois neste há a ideia de conhecimento e educação, não de segurança.

O novo modelo proposto apresentou resultados adequados para todos os critérios: $\mathrm{GFI}=0,913 ; \mathrm{CFI}=0,940$; $\mathrm{CMIN} / \mathrm{DF}=2,617$; e RMSEA = 0,068. Ademais, para validade convergente e discriminante os resultados também foram satisfatórios (Tabela 1).

Tabela 1: Novo modelo proposto, Teresina/Piauí, Brasil, 2016

\begin{tabular}{lllllllllll}
\hline & $\alpha$ & CR & AVE & MSV & ASV & Empatia & Confiabilidade & Presteza & Segurança & Tangibilidade \\
\hline Empatia & 0,839 & 0,848 & 0,53 & 0,482 & 0,419 & 0,728 & & & & \\
Confiabilidade & 0,828 & 0,832 & 0,554 & 0,402 & 0,337 & 0,634 & 0,745 & & & \\
Presteza & 0,779 & 0,782 & 0,545 & 0,45 & 0,395 & 0,658 & 0,624 & 0,738 & & \\
Segurança & 0,716 & 0,717 & 0,559 & 0,45 & 0,344 & 0,599 & 0,544 & 0,671 & 0,748 & \\
Tangibilidade & 0,883 & 0,875 & 0,636 & 0,482 & 0,33 & 0,694 & 0,51 & 0,553 & 0,521 & 0,798 \\
\hline
\end{tabular}

\section{DISCUSSÃO}

Mensurar construtos subjetivos por meio de instrumentos de medida demanda tempo e necessita de uma profunda análise. No caso da satisfação de usuários de serviços de saúde do SUS torna-se mais complexo por envolver várias dimensões da satisfação que englobam desde estrutura física dos serviços de saúde à receptividade dos profissionais de saúde. Neste sentido, há importância em manter em foco a avaliação da qualidade dos serviços prestados por instituições públicas na Atenção Primária à Saúde, especialmente a ESF, gerando um conhecimento que possa balizar as ações e o desenvolvimento de novos programas e estratégias, ou melhorar aqueles já existentes, sendo a avaliação deles componente central para este fim, pois ao avaliar a qualidade dos serviços por meio da satisfação dos usuários é uma forma eficiente na identificação de falhas e contribui para a melhoria da qualidade dos serviços ${ }^{(1,13-16)}$.

O modelo SERVQUAL dispõe de dimensões que abordam questões relacionadas aos fatores da satisfação de usuários que podem ser contextualizados aos serviços de saúde do SUS: Confiabilidade, Tangibilidade, Empatia, Segurança e Presteza ${ }^{(17)}$. Cada dimensão se propõe a mensurar os aspectos relacionados. A Tangibilidade considera desde espaço físico dos serviços à aparência dos profissionais e a situação funcional dos equipamentos disponíveis no serviço de saúde. A Confiabilidade se dedica exclusivamente à relação de confiança entre usuário e profissionais de saúde. A Presteza diz respeito à dedicação do profissional de saúde com o usuário. A Segurança envolve questões simples e mais complexas como o simples ato de esclarecer dúvidas e manutenção do sigilo profissional. A Empatia é o interesse do profissional no usuário em aspectos relacionados à assistência individualizada ${ }^{(8-9,17)}$.

No presente estudo, para o ajuste do modelo, os construtos Presteza e Segurança apresentaram problemas de validade convergente, com valores menores do que 0,5 . Desta forma, sugeriu-se um novo modelo, em que foram retiradas as variáveis P1, P6, P12 e P13. Supõe-se que essas variáveis não se ajustaram ao construto devido aos seus enunciados, a P1 e P6 falam sobre tempo, algo fora do escopo do construto de Confiabilidade, apesar de estarem inseridas em Presteza aparecem aparentemente repetidos em conteúdo em outras variáveis do construto. 
Lago EC, Tapety FI, Almeida CAPL, Matos MCB, Sousa LRM, Martins MCC, et al.

Estudos nacionais apontam queixas de usuários em relação às demoras em atendimentos causadas por longas filas $^{(4)}$. Em contrapartida, estratégias para melhorar o atendimento priorizando a redução do tempo de espera foram implantadas nos últimos anos no SUS, como exemplo o programa "Mais Médicos". O referido programa já apresenta resultados positivos na melhoria da satisfação dos usuários tanto em relação à redução da espera quanto à presteza ${ }^{(18)}$.

Os médicos do programa demonstraram maior dedicação aos usuários por meio da escuta qualificada(18). Resultados semelhantes foram observados em outro estudo(19). Em pesquisa internacional que utilizou o SERVQUAL, houve indicação do tempo de espera para atendimento como uma das lacunas relevantes nos serviços de saúde analisados ${ }^{(14)}$. Considerados problemas recorrentes no sistema único de saúde brasileiro ${ }^{(20)}$. Desta forma, o tempo pode ser considerado uma variável de presteza, reclamada quando o mérito da satisfação dos usuários é investigado. Contudo, não está associado à confiabilidade e pode ser avaliado nas variáveis restante do novo modelo proposto neste estudo.

As variáveis P12 e P13 não se adequaram ao construto Segurança, pois correspondem à ideia de conhecimento e educação, não apresentando relação próxima com a dimensão proposta. Para avaliar a segurança nos serviços de saúde, o comportamento dos funcionários e o sentimento dos usuários em relação ao serviço prestado, as demais variáveis se mostram suficientes no modelo ajustado apresentado.

Estudos indicam que a satisfação relacionada à segurança dos serviços de saúde tem se mostrado baixa em seus aspectos gerais cabendo a gestores o papel de investimentos e incentivos na melhoria da segurança e qualidade da assistência(21).

A segurança do paciente é um assunto abordado de forma ampla pela comunidade científica, com implicações na atuação dos profissionais de saúde para diminuição de riscos e melhoria da segurança com implementação de ações educativas e não punitivas, especialmente para aqueles que se envolvem em eventos que podem comprometer a integridade do paciente ${ }^{(22)}$.

Além de tais ações, os usuários devem perceber o ambiente seguro e se sentirem confortáveis ao solicitar os serviços de saúde para que aumentem os laços de vínculo entre comunidade e profissionais de saúde. Emerge o desafio de tornar o ambiente seguro e repassar boa impressão para os usuários. Elevados níveis de satisfação de usuários dos serviços de saúde, de forma geral, têm relações significativas com a qualidade da saúde das pessoas. Durante o planejamento e implementação de políticas de saúde deve ser levado em consideração à melhoria da satisfação dos usuários ${ }^{(19)}$. Desta forma, o modelo SERVQUAL possibilita a identificação de lacunas na prestação de serviços que podem ser passíveis de preenchimento após análise gerencial da situação, ampliando a percepção de qualidade de serviços e satisfação do consumidor ${ }^{(15,23)}$.

Um estudo conduzido no Irã com pacientes hospitalizados apontou que o construto segurança foi o que obteve resultados mais insatisfatórios podendo afetar a satisfação dos usuários em relação aos serviços prestados $^{(24)}$. Percebe-se que sentir-se seguro em ambientes de cuidado pode ser percebido de forma diferenciada conforme muda o contexto.

Estudos têm sido desenvolvidos na atenção básica brasileira com o objetivo de avaliar a satisfação dos usuários utilizando diferentes abordagens metodológicas. Uma pesquisa de abordagem qualitativa realizada em uma ESF do interior de São Paulo evidenciou que a ESF apresenta evolução quanto à capacidade de acolher e 
Lago EC, Tapety FI, Almeida CAPL, Matos MCB, Sousa LRM, Martins MCC, et al.

resolver problemas da comunidade ${ }^{(25)}$. A consolidação da ESF em diferentes regiões do país aumenta a confiança dos usuários que procuram o serviço, aumenta a procura e a sensação de segurança.

O estudo se limita ao seu objeto, por ter sido desenvolvido em uma ESF. Contudo, com os ajustes do modelo na análise e discussão proposta neste estudo, a realidade de investigação e aplicação da escala pode ser expandida às demais áreas de atuação da ESF e outros serviços de saúde pública ou privada, gerando contribuições a um contexto mais amplo e geral. Além disso, discussões aprofundadas são necessárias para avaliar os itens excluídos da escala neste estudo e, em perspectiva lato, gerar ou consolidar um novo modelo analítico.

\section{CONCLUSÃO}

Como principais resultados, a estrutura dimensional do instrumento mostrou-se adequada mediante o novo modelo proposto ajustado a partir da retirada de algumas variáveis, dado o contexto específico dos serviços de saúde. O estudo implica na indicação de um novo modelo ajustado, utilizado para a compreensão da satisfação de usuários da saúde da família, que, testado em estudos futuros na área de enfermagem e saúde pública, com análise aprofundadas que discutam os achados desta pesquisa, consolidem um novo instrumento analítico, abrindo espaço para comparações futuras com os resultados desta pesquisa.

Os resultados do modelo novo (ajustado), por meio da Análise Fatorial Confirmatória, evidenciaram ser este modelo adequado para uso da mensuração da satisfação dos usuários atendidos no contexto específico da Estratégia Saúde da Família. A abordagem da avaliação da satisfação dos usuários de serviços de saúde, ainda incipiente nos estudos da área, dado o viés analítico predominantemente da gestão organizacional, torna-se uma das principais contribuições do estudo por apresentar uma escala que pode ser convertida em instrumento analítico adequado e resultados que podem ser comparados a outros contextos.

\section{REFERÊNCIAS}

1. Moura LKB, Sousa LRM, Moura MEB, Mesquita RF, Matos FRN, Lago EC. et al. Satisfaction of users of the Family Health Strategy in a capital city of Northeast Brazil. International Archives of Medicine [Internet]. 2017 [acesso em: 19 dez. 2017];10. Disponível em: http://imedicalsociety.org/ojs/index.php/iam/article/view/2407.

2. Brandão ALRBS, Giovanella L, Campos CEA. Avaliação da atenção básica pela perspectiva dos usuários: adaptação do instrumento EUROPEP para grandes centros urbanos brasileiros. Ciênc saúde coletiva [Internet]. 2013 [acesso em: 19 dez. 2017];18(1):103-14. Disponível em: http://www.scielo.br/scielo.php?script=sci_arttext\&pid=S1413-81232013000100012\&lng=en.

3. Stepurko T, Pavlova M, Groot W. Overall satisfaction of health care users with the quality of and access to health care services: a cross-sectional study in six Central and Eastern European countries. BMC Health Serv Res [Internet]. 2016[acesso em: 19 dez. 2017];16(1):342.Disponível em: https://www.ncbi.nlm.nih.gov/pmc/articles/PMC4971706/.

4. Moimaz SAS, Burili MC, Bordin D, Garbin CAS, Saliba TA, Saliba NA. Satisfação dos usuários segundo variáveis de organização dos serviços públicos odontológicos. Arch Health Invest [Internet]. 2017 [acesso em: 8 ago. 2018]; 6(1):14-19. Disponível em: http://www.archhealthinvestigation.com.br/ArcHI/article/view/1776/pdf

5. Alves MM, Leal GS, Sadoyama ADSP, Fernandes EGV, Gomide MDA, Silveira AOSM. et al. O Controle de Infecção Hospitalar como indicador para Qualidade no Serviço de Saúde. Blucher Education Proceedings[Internet]. 2016 [acesso em: 19 dez. 2017];2(1):15872.Disponível em: http://www.proceedings.blucher.com.br/article-details/o-controle-de-infeco-hospitalar-como-indicador-paraqualidade-no-servio-de-sade-25391.

6. Oliaee Z, Jabbari A, Ehsanpour S. An investigation on the quality of midwifery services from the viewpoint of the clients in Isfahan through SERVQUAL model.An investigation on the quality of midwifery services from the viewpoint of the clients in Isfahan through SERVQUAL model.Iran J NursMidwifery Res [Internet]. 2016[acesso em: 19 dez. 2017];21(3):291-6.Disponível em:

https://www.ncbi.nlm.nih.gov/pmc/articles/PMC4857664/. 
7. IBGE [Internet]. Fundação Instituto Brasileiro de Geografia e Estatística. Estatísticas por cidade estado. Fundação Instituto Brasileiro de Geografia e Estatística, dados referentes à cidade de Teresina - Piauí; 2018 [acesso em: 16 out. 2018]. Disponível em: https://www.ibge.gov.br/estatisticas-novoportal/por-cidade-estado-estatisticas.html?t=destaques\&c=2211001.

8. Teshnizi SH, Aghamolaei T, Kahnouji K, Teshnizi SMH, Ghani J. Assessing quality of health services with the SERVQUAL model in Iran. A systematic review and meta-analysis. International Journal for Quality in Health Care [Internet]. 2018 [acesso em: 16 out. 2018], 30(2), 82-89. Disponível em: https://academic.oup.com/intahc/article-abstract/30/2/82/4835623.

9. Al-Neyadi HS, Abdallah S, Malik M. Measuring patient's satisfaction of healthcare services in the UAE hospitals: Using SERVQUAL. International Journal of Healthcare Management [Internet]. 2018 [acesso em: 16 out. 2018], 11(2), 96-105. Disponível em: https://www.tandfonline.com/doi/abs/10.1080/20479700.2016.1266804.

10. Marôco J. Análise Estatística com o SPSS Statistics. 7 ed. Report Number, Lda. 2018.

11. Valentini F, Bruno DF. Variância média extraída e confiabilidade composta: indicadores de precisão. Psicologia: teoria e pesquisa [Internet]. 2017 [acesso em: 16 out. 2018], 32(2):1-7. Disponível em:

https://revistaptp.unb.br/index.php/ptp/article/download/2729/857.

12. Martynova E, West SG, Liu Y. Review of Principles and Practice of Structural Equation Modeling. Structural Equation Modeling: A Multidisciplinary Journal [Internet]. 2018 [acesso em: 16 out. 2018], 25(2):325-329. Disponível em:

https://www.tandfonline.com/doi/full/10.1080/10705511.2017.1401932.

13. Almeida FA, Brito FA. Planejamento e avaliação de programas de saúde: contribuições do Modelo RE-AIM para Enfermagem. Rev Lat Am Enfermagem [Internet]. 2014 [acesso em: 19 dez. 2017];22(4):527-8. Disponível em:

https://www.revistas.usp.br/rlae/article/view/86645/89621.

14.Ogunnowo BE, Olufunlayo TF, Sule SS. Client perception of service quality at the outpatient clinics of a general hospital in Lagos, Nigeria. Pan AfrMed J [Internet]. 2015 [acesso em: 19 dez. 2017];22:68.Disponível em:

https://www.ncbi.nlm.nih.gov/pmc/articles/PMC4725660/.

15. Sousa LRM, Mesquita RF, Matos FRN, Moura LKB, Moura MEB. Dimensões da satisfação dos usuários da Estratégia Saúde da Família. Revista Portuguesa de Investigação Comportamental e Social [Internet].2017 [acesso em: 19 dez. 2017];3(2):2-9. Disponível em: http://dx.doi.org/10.7342/ismt.rpics.2017.3.2.50.

16.Mesquita RF, Sousa MB, MartinsTB, Matos FRN. Óbices metodológicos da prática de pesquisa nas ciências administrativas. RPCA [Internet]. 2014 [acesso em: 19 dez. 2017];8(1):50. Disponível em: http://www.uff.br/pae/index.php/pca/article/view/387/283.

17. Dozinete KC, Medeiros AS, dos Reis ALG, Milan WW, Ferracini MJF. Análise dos cinco gaps pelo método da pesquisa servqual. Revista Eletrônica Organizações e Sociedade [Internet]. 2018 [acesso em: 16 out. 2018], 6(6), 55-68. Disponível em: http://revista.facfama.edu.br/index.php/ROS/article/view/311.

18. Carrapato JFL, Silva RVC, Rotondaro EC, Placideli N. Programa Mais Médicos: percepção dos usuários e dos profissionais do SUS. Revista Em Pauta [Internet]. 2016 [acesso em: 19 dez. 2017];14(38). Disponível em: http://www.e-

publicacoes.uerj.br/index.php/revistaempauta/article/view/27863/20040.

19. Comes Y, Trindade JS, Shimizu HE, Hamann EM, Bargioni F, Ramirez L. et al. Avaliação da satisfação dos usuários e da responsividade dos serviços em municípios inscritos no Programa Mais Médicos. Ciênc saúde coletiva [Internet]. 2016 [acesso em: 19 dez. 2017];21(9):2749-59. Disponível em: http://www.scielo.br/scielo.php?script=sci_arttext\&pid=\$1413-

81232016000902749\&lng=en.

20. Szwarcwald CL, Damacena GN, Souza Júnior PRB, Almeida WS, Malta DC. Percepção da população brasileira sobre a assistência prestada pelo médico. Brasil, 2013. Ciênc saúde coletiva [Internet]. 2016 [acesso em: 19 dez. 2017];21(2):339-50. Disponível em: http://www.scielo.br/scielo.php?script=sci arttext\&pid=S1413-81232016000200339\&lng=en.

21.Bezerra ALQ, Silva TO, Brito TTP, Tobias GC, Silva AEBC. Satisfação dos usuários com a segurança na assistência de enfermagem. Rev enferm UFPE online [Internet].2017 [acesso em: 19 dez. 2017];11(2):915-21. Disponível em:

http://pesquisa.bvs.br/brasil/resource/pt/bde-30863.

22. Oliveira RM, Bandeira ÉDS, Silva CR, Soares AML, Fonteles DB, Barboza FBM. Tomada de decisão de enfermeiros frente a incidentes relacionados à segurança do paciente. Cogitare Enferm [Internet]. 2016[acesso em: 19 dez. 2017];21(3):01-10. Disponível em: http://revistas.ufpr.br/cogitare/article/view/45683/pdf.

23. Purcărea VL, Gheorghe IR, Petrescu CM. The assessment of perceived service quality of public health care services in Romania using the SERVQUAL scale. Procedia Economics and Finance [Internet]. 2013 [acesso em: 19 dez. 2017];6:573-85. Disponível em: http://www.sciencedirect.com/science/article/pii/S2212567113001755.

24. Shafii M, Rafiei S, Abooee F, Bahrami MA, Nouhi M, Lotfi F. et al. Assessment of Service Quality in Teaching Hospitals of Yazd University of Medical Sciences: Using Multi-criteria Decision Making Techniques. Osong Public Health Res Perspect [Internet]. 2016 [acesso em: 19 dez. 2017];7(4):239-47.Disponível em: https://www.ncbi.nlm.nih.gov/pmc/articles/pmid/27635374/.

25. Mishima SM, Campos AC, Matumoto S, Fortuna CM. Satisfação do usuário sob a perspectiva da responsividade: estratégia para análise de sistemas universais? Rev. Latino-Am. Enfermagem [Internet]. 2016 [acesso em: 8 ago. 2018]; 24:e2674. Disponível em: http://www.scielo.br/pdf/rlae/v24/pt 0104-1169-rlae-02674.pdf. 